\title{
Value of a statistical life estimation of carcinogenic chemicals for socioeconomic analysis in Korea
}

\author{
Geonwoo Lee ${ }^{1}$, Yongjin Lee ${ }^{1}$, Hanseul Lee ${ }^{1}$, Jiyeon Hong ${ }^{2,3}$, Jiyeon Yang ${ }^{1}$ \\ ${ }^{1}$ Institute for Environmental Research, Yonsei University Health System, Seoul; ${ }^{2}$ Enterprise Risk Service Deloitte Anjin LLC, \\ Seoul; ${ }^{3}$ Department of Environmental Engineering, Yonsei University, Wonju, Korea
}

\begin{abstract}
Objectives To protect public health from risk, the Minister of Environment in Korea legislated an act concerning the registration and evaluation of chemical substances. In this study, we estimated the value of a statistical life (VSL) of carcinogenic chemicals to evaluate the socioeconomic analysis in Korea.

Methods The estimation of the health benefit can be calculated through an individual's VSL and willingness to pay (WTP). To estimate the VSL and WTP, we used a contingent valuation method through a web-based survey.

Results The survey is conducted with 1434 people living in Seoul and six large cities. An analysis of the survey is essential to review the distribution of the characteristics of the target population. The statistically significant variables affecting the WTP are location, age, household income, quality of life. Through the review of data, we secured statistical validity. The WTP was estimated as 41205 Korean won (KRW)/person, and the estimated VSL appeared as 796 million KRW/person.

Conclusions There is a case in which the amount of statistical life value is estimated in connection with domestic environmental policy, fine dust, etc. However, there are no cases of evaluation for chemical. The utilization of this result is possible for conducting other study with chemicals.
\end{abstract}

Keywords Chemical substance, Contingent valuation method, Socioeconomic analysis, Value of a statistical life, Willingness to pay

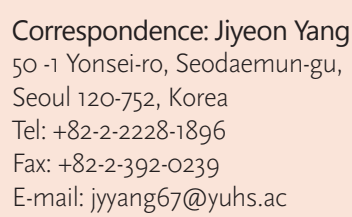

Received: January 8, 2015

Accepted: March 3, 2015

Published online: June 12, 2015

This article is available from: http://e-eht.org/

\section{Introduction}

Industrial chemicals are essential to life in modern society and bring benefits in the form of improved health, food supply, goods, general lifestyle and well-being. The chemical substances that are sold in the domestic market number 43000 or more, and more than 400 species of new chemicals are introduced to the market each year. Some chemicals, if their toxic components enter the environment, can cause problems for human health and ecosystems, and it is important to identify the potential hazardous endpoints, quantify the risk of genuine harm occurring and develop strategies to mitigate that risk.

In the Republic of Korea (hereafter Korea), there are some accidents that cause injury or even death due to chemical sub- stances. To protect the public health from risk, the Miniser of Environment in Korea legislated an Act on the Registration and Evaluation, etc. of Chemical Substances. Socioeconomic analysis (SEA) is used as a basis for determining the safe usage of chemical substances. SEA weighs the costs of any restrictions on the production and use of chemicals against the benefits to human health and the environment. The reasons why industry needs to understand the principles and practices of SEA are: (1) to carry out, where appropriate, an SEA as an argument for authorization, and (2) to be able to contribute as stakeholders to socioeconomic discussions with regulatory authorities when an SEA is used as a basis for justifying restrictions [1]. The evaluation of the SEA needs the technique of benefit estimation, which can be derived from evaluating the value of risk reduction 
and the technique of estimating the potential cost of replacing a chemical with other chemical substances. The estimation of the health benefit can be calculated through an individual's value of a statistical life (VSL) and willingness to pay (WTP) for reducing a risk. There are many techniques for estimating VSL and WTP, and the application of contingent valuation method (CVM) to estimate them has recently been increased [2].

In this study, we used the CVM to estimate a WTP for reducing a mortality risk.

\section{Materials and Methods}

To estimate the health benefit, we evaluated VSL and WTP using web-based survey methods and used a double-bounded dichotomous choice (DBDC), which is known for having a small bias. The DBDC method, in order to reduce the risk of chemicals, is a form asking whether the participant is willing to pay an amount of money. In particular, the DBDC method is widely used more than the single-bounded dichotomous choice that asks only one question [3]. The DBDC question is presented in the form of two amounts to each respondent [4]. First, we requested a response of "yes" or "no" as to whether they would pay more than the amount of their first proposed WTP. The amount of the second presentation depended on the figure proposed in the first presentation (Figure S1).

We developed the survey program based on the DBDC that is utilized in resources for the future. The survey is conducted with 1434 people living in Seoul and six large cities (Gwangju, Daejeon, Incheon, Daegu, Busan, Ulsan). One-to-one interviews take more than a month, but they can be completed within one week when performing a web-based survey of 1000 people. After completion of the survey, the results are applied to the algorithm of VSL calculation. The lists to calculate VSL include: the distribution of residence in a large city, age, sex, health status compared with the same age group, the satisfaction with health after 10 years, current disease status, family history, family income, number of family, marriage, treatment for and survival of cancer, smoking status, housing type, last educational background, job status, and amount willing to pay to reduce risks. The analysis of each list is important to ensure reliability of results.

\section{Results}

The web-based questionnaire was performed using four structure of DBDC, randomly. The structure changes depending on the suggested payable amount first proposed. The distributions of the results of the analyzed payable amounts and characteristics can be determined as reliable data. We conducted a questionnaire survey using the initial amounts of 4000 Korean won
(KRW), 10000 KRW, 20000 KRW, and 40000 KRW (Table 1).

An analysis of the survey is essential to review the distribution of the characteristics of the target population in order to apply the variables for estimating the VSL. The results are as follows. The survey involved 721 males and 713 females, aged 30 to 69 $(\mathrm{n}=419), 40$ to $49(\mathrm{n}=438), 50$ to $59(\mathrm{n}=379)$ and 60 to 69 $(n=198)$. Distribution of the residence of the respondents was highest (44\%) in Seoul, 15\% in Busan, 12\% in Incheon, 11\% in Daegu, 7\% in Daejeon, 6\% in Gwangju, and 5\% in Ulsan. The distribution of surveyed residence was suitable for estimation of the VSL nationwide. The highest response regarding satisfaction of health status (very bad, bad, normal, good, very good) was good ( $30 \%$ to $40 \%$ ). The responses of very bad, bad, very good were 0.5 $\%$ to $3.5 \%, 13.1 \%$ to $20.0 \%, 3.2 \%$ to $6.7 \%$, respectively. The results of the survey on health status after 10 years showed that the current health status will similar to that of after 10 years. The analysis of health status is important in relation to the amounts participants are willing to pay. Diseases diagnosed among participants in the different regions were: hypertension, gastric ulcer and duodenal disease, liver disease, diabetes, thyroid disease, chronic respiratory disease, heart disease, cancer, cerebrovascular disease, hyperlipidemia, and renal failure were analyzed in order, in Seoul. In Busan, hypertension, gastric ulcer and duodenal diseases, liver disease, diabetes, heart disease, thyroid disease, chronic respiratory disease, and cancer were analyzed in order. Other areas were similarly analyzed by disease diagnosis, and the bias of participants living in other cities can be considered small. The response to anticipated life-expectancy was 71 years to 80 years (41.9\%), 81 years to 90 years (38\%). The results of smoking status show that the percentage of smokers was about $22 \%$, about $16 \%$ were exsmokers, and $62 \%$ were non-smokers. The distributions of housing type were $65 \%$ owner occupation, $25 \%$ annual rent, and $8 \%$ monthly rent. Regarding educational background, in Seoul less than $3 \%$ were educated to middle school, $22 \%$ to high school, $12 \%$ went to professional college, $52 \%$ were college graduates, and $11 \%$ were to graduate school. Regionally, while there are differences in the percentages, the results are similar. In the case of white-collar workers includes more people than any other type of job (30.4\%). The ranges of payable amounts were separated as follows: $0 \mathrm{KRW}$

Table 1. The structure of suggested amounts for conducting doublebounded dichotomous choice

\begin{tabular}{lccc}
\hline \multirow{2}{*}{ Type of survey } & Initial amount & \multicolumn{2}{c}{ Second amount } \\
\cline { 3 - 4 } & & If, yes & If, no or not sure \\
\hline A & 4000 & 10000 & 2000 \\
B & 10000 & 20000 & 4000 \\
C & 20000 & 40000 & 10000 \\
D & 40000 & 70000 & 20000 \\
\hline
\end{tabular}

Unit: Korean won. 


\begin{tabular}{lcccc}
\hline \multirow{2}{*}{ Study } & \multicolumn{2}{c}{ Risk of death (type) } & \multicolumn{2}{c}{ VSL (95\% Cl) } \\
\cline { 2 - 4 } & Death & Factors & Form of WTP & 796 (392, 2215) \\
\hline This study & Cancer & - & Product & $321(160,642)$ \\
Shin [5] & Cancer & - & Product & $1480(1128,1833)$ \\
Shin [6] & All & - & Product & $356(315,430)$ \\
Lee et al. [7] & Cancer & - & Product & $287(233,311)$ \\
Kim et al. [8] & All & PM & Product & $466(339,594)$ \\
Shin et al. [9] & All & Policy & Product & $1149(243,2314)$ \\
Shin [10] & All & - & Product & \\
\hline
\end{tabular}

Unit: million Korean won.

VSL, value of a statistical life; WTP, willingness to pay; Ci, confidence interval; PM, particulate matter.

to $5000 \mathrm{KRW}, 5000 \mathrm{KRW}$ to $10000 \mathrm{KRW}, 10000 \mathrm{KRW}$ to 50000 KRW, $50000 \mathrm{KRW}$ to $100000 \mathrm{KRW}$, and $100000 \mathrm{KRW}$ to 150000 KRW. The percentages of participants in the A type were $21 \%, 23.9 \%, 47.2 \%, 7.2$, and $8.2 \%$, respectively; in B type $25.5 \%$, $24.7 \%, 77.8 \%, 6.6 \%$, and $0.8 \%$, respectively; in C type were $25.2 \%$, $25.1 \%, 42.1 \%, 6.9 \%$, and $0.8 \%$, respectively. $25.6 \%, 24.1 \%$, $44.2 \%$, 6.0 , and $0.2 \%$, respectively in D type. The highest range of average amounts of the 1434 survey participants was $10000 \mathrm{KRW}$ to $50000 \mathrm{KRW}$, there were no significant differences between the overall average results of the 1434 people surveyed and results were separated according to type. In order to estimate the VSL for chemicals in Korea, we applied various data for statistical validity. The final model for calculating WTP was built by lifereg plus maximum iteration. The statistically significant variables affecting the WTP are location, age (square), household income, quality of life. The beta coefficient of location, age (square), household income, and quality of life was $0.33,-0.0002,0.09$, and 0.16 , respectively.

\section{Discussion}

In the analysis of the questionnaire results, the mean WTP for the 5 , in 10000 risk reduction is statistically greater than the mean WTP for the 1 in 10000 risk reduction. As a result, we derived the amounts of WTP and individual's VSL for the 5 in 10000 risk reduction. The WTP was estimated at $41205 \mathrm{KRW} /$ person, and the estimated VSL appeared as 796 million KRW/person. The standard error was 172 million KRW, 95\% lower confidence limit was 392 million KRW, upper confidence limit was 2215 million KRW. There are studies for calculating the VSL in Korea. We compared the results of other studies (Table 2).

When we compared the VSL of this study with another major domestic study, the estimated amounts appear to be slightly higher in ours. But our amounts are low compared to the results of other countries. There is a case in which the amount of statistical life value was estimated in connection with domestic envi- ronmental policy, fine dust, etc. However, there are no cases evaluating a chemical. In conjunction with the progression of similar studies, utilization of these results is possible, and we will compare the results of similar studies and methods, and confirm the validity of the technique for surveying and calculating VSL.

\section{Acknowledgements}

This study was supported by the Korea Ministry of Environment (MOE) as "MOE's R\&D Program on environmental technology development" (no. 412-111-005).

\section{Conflict of Interest}

The authors have no conflicts of interest with material presented in this paper.

\section{References}

1. European Centre for Ecotoxicology and Toxicology of Chemicals. Environmental impact assessment for socio-economic analysis of chemicals: principles and practice. Brussels: European Centre for Ecotoxicology and Toxicology of Chemicals; 2011, p. 3-4.

2. Hammitt JK, Graham JD. Willingness to pay for health protection: inadequate sensitivity to probability? J Risk Uncertain 1999;18(1):3362.

3. Lee YJ, Lim YW, Yang JY, Kim CS, Shin YC, Shin DC. Evaluating the PM damage cost due to urban air pollution and vehicle emissions in Seoul, Korea. J Environ Manage 2011;92(3):603-609.

4. Kim YS, Lee YJ, Park HS, Nam CM, Kim JH, Shin DC. Estimation of willingness to pay for reduction of environmental mortality risk. J Environ Toxicol 2003;18(1):1-13 (Korean).

5. Shin YS. Estimating the value of statistical life by analysing disease protective behavior: focusing on medical examination of cancer. Environ Resour Econ Rev 2008;17(4):845-873 (Korean).

6. Shin YC. Estimating values of statistical lives using choice experiment method. Environ Resour Econ Rev 2007;16(3):683-702 (Ko rean). 
7. Lee YJ, Kim YS, Shin DC, Shin YC. A study on developing a model for cancer damage cost due to risk from benzene in Ulsan metropolitan city. Environ Resour Econ Rev 2004;13(1):49-82 (Korean).

8. Kim YS, Lee YJ, Park HS, Shin DC. Risk-based damage cost estimation on mortality due to environmental problems. Korean J Prev Med 2003;36(3):230-238 (Korean).
9. Shin YC, Joh SH. Estimating the willingness-to-pay and the value of a statistical life for future nortality risk reduction: the value of a statistical life for assessing environmental damages and policies. Environ Resour Econ Rev 2003;12(1):49-74 (Korean).

10. Shin YS. Estimating the cost of air pollution on morbidity: focusing on hospital visit for acute respiratory diseases. Environ Resour Econ Rev 2002;11(4):659-687 (Korean). 


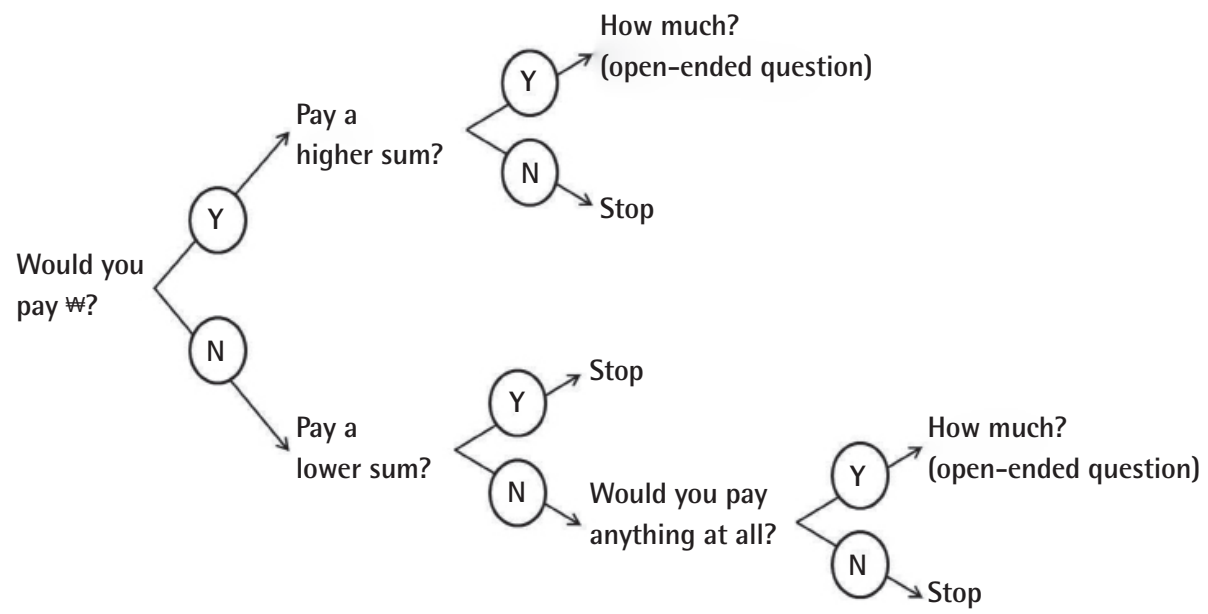

Figure S1. Double-bounded dichotomous choice methods. From Lee YJ, et al. J Environ Manage 2011;92:603-609 [3]. Y, yes; N, no. 